\title{
Convex Functions on Discrete Sets
}

\author{
Christer O. Kiselman \\ Uppsala University, P. O. Box 480, SE-75106 Uppsala, Sweden \\ kiselman@math.uu.se \\ http://www.math.uu.se/ ${ }^{k i s e l m a n}$
}

\begin{abstract}
We propose definitions of digital convex sets and digital convex functions and relate them to a refined definition of digital hyperplanes.
\end{abstract}

Keywords: Digital convex set, digital convex function, naive digital line, digital hyperplane, Fenchel transformation.

\section{Introduction}

Digital geometry is a branch of geometry which is inspired by the use of computers in creating images and of importance for the proper understanding and creation of many algorithms in image processing. In Euclidean geometry convex sets play an important role, and convex functions of real variables are of importance in several branches of mathematics, especially in optimization.

All this forms the background of the present paper, where we will propose definitions of convex sets (Definition 3.1) and convex functions (Definition 4.1) in a digital setting, definitions that have many desirable properties. They are in fact very simple - some may call them naive - but it seems to be necessary to investigate them first before one can go on to more sophisticated definitions. We shall show that functions which are both convex and concave have interesting relations to a refined definition of digital hyperplanes.

The notion of a digital straight line received a satisfying definition in Rosenfeld's seminal paper (1974), where he explained how to digitize a real straight line segment. Since then, variants of this digitization have been introduced, among them digitizations which respect the Khalimsky topology; see Melin (2003). Here we shall not consider the Khalimsky topology, however. Instead, we shall look at definitions of digital hyperplanes, in particular that of Reveillès (1991), and compare them with the notion of digitally convex and concave functions.

We cannot mention here all the work done on convex sets and digital planes; we refer the reader to the surveys by Eckhardt (2001) and Rosenfeld \& Klette (2001) and the many papers referred to there. Eckhardt studies no less than five different notions of convexity; one of them he calls H-convexity (2001:218) — this is the notion studied in the present paper.

We write $\mathbf{Z}$ for the ring of integers and $\mathbf{R}$ for the field of real numbers. When defining functions with integer values, we shall often use the floor and ceiling functions $\mathbf{R} \ni t \mapsto\lfloor t\rfloor,\lceil t\rceil \in \mathbf{Z}$. They are uniquely determined by the 
requirement that $\lfloor t\rfloor$ and $\lceil t\rceil$ be integers for every real number $t$ and by the inequalities

$$
t-1<\lfloor t\rfloor \leqslant t<\lfloor t\rfloor+1 ; \quad\lceil t\rceil-1<t \leqslant\lceil t\rceil<t+1, \quad t \in \mathbf{R} .
$$

\section{The Real Case}

Let $E$ be a vector space over the field of real numbers. A subset $A$ of $E$ is said to be convex if the segment $[a, b]=\{(1-t) a+t b ; 0 \leqslant t \leqslant 1\}$ is contained in $A$ for every choice of $a, b \in A$; in other words if $\{a, b\} \subset A$ implies $[a, b] \subset A$. And convex functions are most conveniently defined in terms of convex sets: a function $u: E \rightarrow[-\infty,+\infty]=\mathbf{R} \cup\{+\infty,-\infty\}$ is said to be convex if its epigraph

$$
\text { epi } u=\{(x, t) \in E \times \mathbf{R} ; u(x) \leqslant t\}
$$

is a convex set in $E \times \mathbf{R}$. For functions $f: P \rightarrow[-\infty,+\infty]_{\mathbf{Z}}=\mathbf{Z} \cup\{+\infty,-\infty\}$, where $P$ is a subset of $E$, we define the epigraph as a subset of $P \times \mathbf{Z}$ :

$$
\text { epi } f=\{(p, q) \in P \times \mathbf{Z} ; f(p) \leqslant q\} .
$$

We shall also need the strict epigraph of $u$, which is the set

$$
\operatorname{epi}_{\mathrm{s}} u=\{(x, t) \in E \times \mathbf{R} ; u(x)<t\} .
$$

It is convex if and only if $u$ is convex.

Related to these notions are the graph and hypograph of a function, defined by $\operatorname{graph} u=\{(x, t) \in E \times \mathbf{R} ; u(x)=t\}$ and hypo $u=\{(x, t) \in E \times \mathbf{R} ; u(x) \geqslant t\}$, respectively.

It is also possible to go in the other direction and define convex sets in terms of convex functions: a set $A$ in $E$ is convex if and only if its indicator function $i_{A}$ is convex, where we define $i_{A}(x)=0$ if $x \in A$ and $i_{A}(x)=+\infty$ otherwise. Naturally we would like to keep these equivalences in the digital case.

Important properties of the family of convex sets in a vector space are the following.

Proposition 2.1. If $C_{j}, j \in J$, are convex sets, then the intersection $\bigcap C_{j}$ is convex. If the index set $J$ is ordered and filtering to the right, and if $\left(C_{j}\right)_{j \in J}$ is an increasing family of convex sets, then its union $\bigcup C_{j}$ is convex.

Because of this result, the intersection

$$
\operatorname{cvx} A=\bigcap(C \in \mathscr{P}(E) ; C \text { is convex and } C \supset A), \quad A \in \mathscr{P}(E),
$$

of all convex sets containing a given subset $A$ of $E$ is itself convex; it is called the convex hull of $A$.

Proposition 2.2. If $u_{j}, j \in J$, are convex functions on a vector space, then $\sup u_{j}$ is convex. If the index set $J$ is ordered and filtering to the right, and if 
$\left(u_{j}\right)_{j \in J}$ is a decreasing family of convex functions, then its infimum inf $u_{j}$ is convex.

To a given function $u: E \rightarrow[-\infty,+\infty]$ we associate two convex functions, viz. the supremum $v$ of all convex minorants of $u$ and the supremum $w$ of all affine minorants of $u$. According to Proposition 2.2 these functions are themselves convex, and of course $w \leqslant v \leqslant u$. We shall denote $v$ by $\operatorname{cvx} u$, and $w$ by $\widetilde{\widetilde{u}}$, a notation which will become clear when we have introduced the Fenchel transformation below. The function $v=\operatorname{cvx} u$ will be called the convex hull of $u$. For functions $f: P \rightarrow[-\infty,+\infty], P$ being a subset of $E$, we shall use the same notation. Such a function can be extended to a function $u$ defined in all of $E$ simply by taking $u=+\infty$ in the complement of $P$ (then $u$ and $f$ have the same epigraph), and we define $\operatorname{cvx} f=\operatorname{cvx} u$.

In many cases, but not always, $\widetilde{\widetilde{u}}$ is equal to $\operatorname{cvx} u$ (see Examples 2.3 and 2.4 below). To understand this, we note that $\widetilde{\widetilde{u}}$ has two extra properties in addition to being convex, properties that are not always shared by cvx $u$. The first is that $\widetilde{\widetilde{u}}$ is lower semicontinuous for any topology for which the affine functions are continuous. The second is that if $u$ takes the value $-\infty$ at a point, then $\widetilde{\widetilde{u}}$ must be identically equal to $-\infty$ (there are no affine minorants), whereas cvx $u$ may take also finite values or $+\infty$.

Example 2.3. Let $P \subset \mathbf{R}^{2}$ be the set of all points $\left(p_{1}, 1 /\left(1+p_{1}^{2}\right)\right), p_{1} \in \mathbf{Z}$. Define a function $f: P \rightarrow[0,+\infty]$ by $f(p)=0$ for $p \in P$. Then $\operatorname{cvx} f$ takes the value 0 when $0<x_{2}<1$ or $x=(0,1)$. On the other hand $\widetilde{\widetilde{f}}$ takes the value 0 in the closed strip $0 \leqslant x_{2} \leqslant 1$ and $+\infty$ elsewhere, so the two functions differ when $x_{2}=0$ or $x_{2}=1, x_{1} \neq 0$.

Example 2.4. Let $\alpha$ be an irrational number and define $f: \mathbf{Z}^{2} \rightarrow[0,+\infty]$ by $f(p)=0$ if $p_{2}=\left\lceil\alpha p_{1}\right\rceil, p_{1} \neq 0 ; f(0)=3$; and $f(p)=+\infty$ otherwise. Then $(\operatorname{cvx} f)(x)=0$ if $x$ is in the open strip $\alpha x_{1}<x_{2}<\alpha x_{1}+1 ;(\operatorname{cvx} f)(0)=3$; and $(\operatorname{cvx} f)(x)=+\infty$ elsewhere. On the other hand $\widetilde{\widetilde{f}}(x)=0$ when $\alpha x_{1} \leqslant x_{2} \leqslant$ $\alpha x_{1}+1$ and $+\infty$ outside this closed strip.

We thus have

$$
w=\widetilde{\widetilde{u}} \leqslant v=\operatorname{cvx} u \leqslant u .
$$

However, in our research it will not be enough to study these functions: it is necessary to look at their epigraphs.

The epigraph epi $u$ of $u$ is a subset of $E \times \mathbf{R}$ and its convex hull $C=\operatorname{cvx}($ epi $u)$ is easily seen to have the property

$$
(x, s) \in C, s \leqslant t \text { implies }(x, t) \in C .
$$

The function $V_{C}(x)=\inf (t ;(x, t) \in C)$ satisfies

$$
\operatorname{epi}_{\mathrm{s}} V_{C} \subset \operatorname{cvx}(\text { epi } u) \subset \text { epi } V_{C} .
$$

It is clear that $V_{C}$ is convex and equal to the largest convex minorant $v=\operatorname{cvx} u$ of $u$ already introduced. Thus $\operatorname{cvx} u$ can be retrieved from $\operatorname{cvx}(\operatorname{epi} u)$ but not 
conversely. The inequality (2.1) and the inclusion relation (2.3) can be combined to

$$
\operatorname{epi}_{\mathrm{s}} u \subset \operatorname{epi}_{\mathrm{s}}(\operatorname{cvx} u) \subset \operatorname{epi}_{\mathrm{s}}(\operatorname{cvx} u) \cup \operatorname{epi} u \subset \operatorname{cvx}(\operatorname{epi} u) \subset \operatorname{epi}(\operatorname{cvx} u) \subset \operatorname{epi} \widetilde{\widetilde{u}},
$$

and in general we cannot claim that cvx (epi $u$ ) is an epigraph (see Examples 4.2 and 4.3).

In this paper, convex sets which are squeezed in between the epigraph and the strict epigraph of a function will play an important role. Such sets $C$ satisfy $\operatorname{epi}_{\mathrm{s}} u \subset C \subset$ epi $u$ for some function $u$. This means that $C$ is obtained from the strict epigraph by adding some points in the graph:

$$
C=\operatorname{epi}_{\mathrm{s}} u \cup\{(x, u(x)) ; x \in A\} \subset \operatorname{epi}_{\mathrm{s}} u \cup \operatorname{graph} u=\text { epi } u .
$$

Extreme examples are the following. If $u$ is strictly convex, like $u(x)=\|x\|_{2}^{p}$, $x \in \mathbf{R}^{n}$, with $1<p<+\infty$, then any such set is convex, even though $A$ may be very irregular. If on the other hand $u=0$, then such a set is convex if and only if $A$ itself is convex.

Definition 2.5. Let $E$ be a real vector space and denote by $E^{\star}$ its algebraic dual (the set of all real-valued linear forms on $E$ ). For any function $u: E \rightarrow[-\infty,+\infty]$ we define its Fenchel transform $\widetilde{u}$ by

$$
\widetilde{u}(\xi)=\sup _{x \in E}(\xi(x)-u(x)), \quad \xi \in E^{\star} .
$$

For any function $v: F \rightarrow[-\infty,+\infty]$ defined on a vector subspace $F$ of $E^{\star}$ we define its Fenchel transform by

$$
\widetilde{v}(x)=\sup _{\xi \in F}(\xi(x)-v(\xi)), \quad x \in E .
$$

The second Fenchel transform $\widetilde{\widetilde{u}}$ of $u$ is well-defined if we fix a subspace $F$ of $E^{\star}$. This subspace can be anything between $\{0\}$ and all of $E^{\star}$, in particular we can take $F$ as the topological dual $E^{\prime}$ of $E$ if $E$ is equipped with a vector space topology.

The restriction $\left.\widetilde{u}\right|_{F}$ of the Fenchel transform to a subspace $F$ of $E^{\star}$ describes all affine minorants of $u$ with linear part in $F$ : a pair $(\xi, \beta) \in F \times \mathbf{R}$ belongs to epi $\widetilde{u}$ if and only if $x \mapsto \xi(x)-\beta$ is a minorant of $u$. This implies that $\widetilde{\widetilde{u}}$ is the supremum of all affine minorants of $u$ with linear part in $F$. This function is a convex minorant of $u$, but it has the additional properties that it cannot take the value $-\infty$ unless it is the constant $-\infty$, and it is lower semicontinuous with respect to the topology $\sigma(E, F)$, the weakest topology on $E$ for which all linear forms in $F$ are continuous. One can prove that $\widetilde{\widetilde{u}}$ is the largest convex minorant of $u$ with these properties. General references for the Fenchel transformation are Hörmander (1994), Singer (1997) and Hiriart-Urruty \& Lemaréchal (2001). 


\section{Convex Sets}

Definition 3.1. Let $E$ be a real vector space and fix a subset $P$ of $E$. A subset $A$ of $P$ is said to be $P$-convex if there exists a convex set $C$ in $E$ such that $A=C \cap P$.

We are mostly interested in the case $E=\mathbf{R}^{n}, P=\mathbf{Z}^{n}$.

For digitizations of convex sets the mapping $C \mapsto C \cap \mathbf{Z}^{n}$ is not always satisfactory, because it yields the empty set for some long and narrow convex sets $C$. One might then want to replace it by a mapping like $C \mapsto(C+B) \cap \mathbf{Z}^{n}$, where $B$ is some fixed set which guarantees that the image is nonempty when $C$ is nonempty, e.g., $B=B_{\leqslant}(0, r)$, where $r=1 / 2$ if we use the $l^{\infty}$ norm in $\mathbf{R}^{n}$, $r=\sqrt{n} / 2$ if we use the $l^{2}$ norm, or $r=n / 2$ if we use the $l^{1}$ norm. However, for our purpose, when we apply this operation to the epigraph of a function, this phenomenon will not appear: the epigraph of a function with finite values always intersects $\mathbf{Z}^{n} \times \mathbf{Z}$ in a nonempty set.

Lemma 3.2. Given a vector space $E$ and a subset $P$ of $E$, the following properties are equivalent for any subset $A$ of $P$.

1. A is P-convex;

2. $A=(\operatorname{cvx} A) \cap P$;

3. $A \supset(\operatorname{cvx} A) \cap P$.

4. For all $n$, all $a_{0}, \ldots, a_{n} \in A$, and for all nonnegative numbers $\lambda_{0}, \ldots, \lambda_{n}$ with $\sum_{0}^{n} \lambda_{j}=1$, if $\sum_{0}^{n} \lambda_{j} a_{j} \in P$, then $\sum_{0}^{n} \lambda_{j} a_{j} \in A$.

Proof. This is easy. As far as property 4 is concerned, we can, in view of Carathéodory's theorem, let $n$ be the dimension of $E$ if the space is finite dimensional; otherwise we must use all $n$.

Definition 3.3. Fix two subsets $P$ and $Q$ of a vector space $E$ and define an operator $\gamma=\gamma_{P, Q}: \mathscr{P}(E) \rightarrow \mathscr{P}(P)$ by $\gamma(A)=\operatorname{cvx}(A \cap Q) \cap P$.

We can think of $E=\mathbf{R}^{n}, P=m \mathbf{Z}^{n}, m=1,2, \ldots$, and $Q=\mathbf{Z}^{n}$. We note that $\gamma(C)$ is $P$-convex if $C$ is convex in $\mathbf{R}^{n}$.

Lemma 3.4. The mapping $\gamma$ is increasing; it satisfies $\gamma(\gamma(A)) \subset \gamma(A)$; and it satisfies $A \subset \gamma(A)$ if $A \subset P \cap Q$. Thus $\left.\gamma\right|_{\mathscr{P}(P)}$ is a closure operator in $\mathscr{P}(P)$ if $Q \supset P$.

Proof. The mapping $\gamma=j_{P} \circ \mathrm{cvx} \circ j_{Q}$ is a composition of three increasing mappings, viz. $j_{Q}$ (intersection with $Q$ ), cvx (taking the convex hull), and $j_{P}$ (intersection with $P$ ), and as such itself increasing. The composition $\gamma \circ \gamma$ is equal to $j_{P} \circ \mathrm{cvx} \circ j_{Q} \circ j_{P} \circ \mathrm{cvx} \circ j_{Q}$, which is smaller than $j_{P} \circ \mathrm{cvx} \circ \mathrm{cvx} \circ j_{Q}=$ $j_{P} \circ \mathrm{cvx} \circ j_{Q}=\gamma$. Finally, it is clear that $\gamma(A)$ contains $A$ if $A$ is contained in $P \cap Q$. If $Q \supset P$, then $\gamma$ is increasing, idempotent and extensive, thus a closure operator in $\mathscr{P}(P)$. 
Proposition 3.5. Let $E$ be a real vector space and $P$ any subset of $E$. Then $A$ is $P$-convex iff $A=\gamma(A)$ for all $Q \supset P$ iff $A=\gamma(A)$ for some $Q \supset P$.

Proof. If $A$ is $P$-convex, $A=C \cap P$, then $\gamma(A)=\gamma(C \cap P)=\operatorname{cvx}(C \cap P \cap Q) \cap P=$ $C \cap P=A$ for all $Q \supset P$.

If $A=\gamma(A)$ for some choice of $Q \supset P$, then $A=\operatorname{cvx}(A \cap Q) \cap P=C \cap P$ if we define $C=\operatorname{cvx}(A \cap Q)$, so that $A$ is $P$-convex.

Corollary 3.6. If $A=C \cap P$, then $C \supset \gamma(A)$ for any $Q$.

Thus in the definition of $P$-convex sets we may always take $C=\gamma(A)=\operatorname{cvx} A$ provided $Q \supset P$.

It is now easy to prove the following result.

Proposition 3.7. Let $E$ be a vector space and $P$ any subset of $E$. If $A_{j}, j \in J$, are $P$-convex sets, then the intersection $\bigcap A_{j}$ is $P$-convex. If the index set $J$ is ordered and filtering to the right, and if $\left(A_{j}\right)_{j \in J}$ is an increasing family of $P$-convex sets, then its union $\bigcup A_{j}$ is also $P$-convex.

Proof. For each $A_{j}$ we have $A_{j}=C_{j} \cap P$, where $C_{j}=\operatorname{cvx} A_{j}$ is a convex set in $E$. Then $\bigcap A_{j}=\bigcap\left(C_{j} \cap P\right)=\left(\bigcap C_{j}\right) \cap P$. The last set is $P$-convex in view of Proposition 2.1.

For the union we have $\bigcup A_{j}=\bigcup\left(C_{j} \cap P\right)=\left(\bigcup C_{j}\right) \cap P$, so Proposition 2.1 gives also the second statement - the family $\left(C_{j}\right)=\left(\operatorname{cvx} A_{j}\right)$ is increasing since $\left(A_{j}\right)$ is.

While the intersection of two $P$-convex epigraphs gives a reasonable result, the intersection of an epigraph and a hypograph may consist of two points quite far from each other:

Example 3.8. Let $A=\left\{p \in \mathbf{Z}^{2} ; p_{2} \geqslant p_{1} / m\right\}$ and $B=\left\{p \in \mathbf{Z}^{2} ; p_{2} \leqslant p_{1} / m\right\}$, where $m \in \mathbf{N} \backslash\{0\}$. Then $A$ and $B$ are $\mathbf{Z}^{2}$-convex and their intersection consists of all points $\left(m p_{2}, p_{2}\right), p_{2} \in \mathbf{Z}$. We can easily modify the example so that the intersection consists of exactly two points, $(0,0)$ and $(m, 1)$, where $m$ is as large as we please.

\section{Convex Functions}

Definition 4.1. Let $E$ be a vector space and $P$ any of its subsets. A function $f: P \rightarrow[-\infty,+\infty]_{\mathbf{Z}}$ is said to be $(P \times \mathbf{Z})$-convex if its epigraph

$$
\text { epi } f=\{(p, t) \in P \times \mathbf{Z} ; f(p) \leqslant t\}
$$

is a $(P \times \mathbf{Z})$-convex subset of $E \times \mathbf{R}$.

We have mainly the case $E=\mathbf{R}^{n}$ and $P=\mathbf{Z}^{n}$ in mind.

If $f: P \rightarrow[-\infty,+\infty]_{\mathbf{z}}$ is a $P$-convex function, then there is a convex set $C$ in $E \times \mathbf{R}$ such that $C \cap(P \times \mathbf{Z})=$ epi $f$. In view of Corollary 3.6, the smallest such 
set $C$ is the convex hull of epi $f$. However, a set $C$ such that $C \cap(P \times \mathbf{Z})=$ epi $f$ does not necessarily have the property $(2.2)$, so we introduce

$$
C^{+}=\{(x, t) \in E \times \mathbf{R} ; \exists s \leqslant t \text { with }(x, s) \in C\} .
$$

There is a function $V_{C^{+}}: E \rightarrow[-\infty,+\infty]$ such that

$$
\text { epi }_{\mathrm{s}} V_{C^{+}} \subset C^{+} \subset \operatorname{epi} V_{C^{+}} \text {. }
$$

It would perhaps seem natural to require that $C^{+}$be closed or open so that one could always take either the epigraph or the strict epigraph of $V_{C^{+}}$, but simple examples (see below) show that this is not possible. We note that when we take $C=\operatorname{cvx}($ epi $f)$, then $C^{+}=C$.

Some care is needed, because even if epi $f$ is closed, its convex hull need not be closed:

Example 4.2. Let $f_{0}(p)=\lceil\alpha p\rceil, p \in \mathbf{Z}$, where $\alpha$ is irrational. We also define $f_{1}(p)=f_{0}(p)$ for $p \in \mathbf{Z} \backslash\{0\}$ and $f_{1}(0)=1$. These functions are easily seen to be $(\mathbf{Z} \times \mathbf{Z})$-convex. Indeed, cvx (epi $\left.f_{1}\right)$ is the open half plane $C_{1}=\{(x, t) ; t>\alpha x\}$, a strict epigraph, and cvx (epi $\left.f_{0}\right)$ is the convex set $C_{0}=C_{1} \cup\{(0,0)\}$, which is neither an epigraph nor a strict epigraph. (However, also the closed half plane $\{(x, t) ; t \geqslant \alpha x\}$ intersects $\mathbf{Z}^{2}$ in epi $f_{0}$.) We finally note that the functions $-f_{0}$ and $-f_{1}$ are $(\mathbf{Z} \times \mathbf{Z})$-convex as well.

A convex function need not be determined by its restriction to the complement of a point: in Example 4.2 above, $f_{0}$ and $f_{1}$ agree on $\mathbf{Z} \backslash\{0\}$. This kind of ambiguity is, however, something we have to live with if we want results like Proposition 2.2 to hold. In the example $f_{0}$ is a supremum of convex functions without this ambiguity, and $f_{1}$ is the limit of a decreasing sequence of convex functions without the ambiguity. To make this precise, define $g_{s}(p)=\lceil\alpha p+s\rceil$, $p \in \mathbf{Z}$, where $s$ is a real parameter. Then $g_{s} \rightarrow f_{1}$ as $s$ tends to zero through positive values, and $g_{s} \rightarrow f_{0}$ as $s$ tends to zero through negative values. We have $g_{s}(0)=1$ for $0<s<1$, and we claim that $g_{s}(0)$ is determined by the restriction of $g_{s}$ to the complement of the origin when $0<s<1$. Indeed, let an extension take the value $c \in \mathbf{Z}$ at the origin. Then $c \leqslant 0$ is impossible for $s>0$, and $c \geqslant 2$ is impossible for all $s$ such that $0<s<1$. Similarly $g_{s}$ with $s$ negative is determined from its restriction to $\mathbf{Z} \backslash\{0\}$. So the functions $g_{s}$ with $s$ small and nonzero do not have this kind of ambiguity, whereas their limits as $s \rightarrow 0 \pm$ do.

Example 4.3. Let a set $A$ of even integers be given and define $g_{A}(p)=\left\lceil\frac{1}{2} p\right\rceil=$ $\frac{1}{2} p+\frac{1}{2}, p \in \mathbf{Z}, p$ odd, and $g_{A}(p)=\frac{1}{2} p$ when $p$ is even, $p \in A$, and $g_{A}(p)=\frac{1}{2} p+1$ when $p$ is even and $p \notin A$. This function is $(\mathbf{Z} \times \mathbf{Z})$-convex if and only if $A$ is an interval in $2 \mathbf{Z}$. To see this, we note that $\operatorname{cvx}\left(\operatorname{epi} g_{A}\right)$ is the convex set

$$
C_{I}=\left\{(x, t) \in \mathbf{R} \times \mathbf{R} ; t>\frac{1}{2} x\right\} \cup\left\{\left(x, \frac{1}{2} x\right) \in I \times \mathbf{R}\right\},
$$

where $I$ is the convex hull of $A$. Then $C_{I} \cap(\mathbf{Z} \times \mathbf{Z})$ is equal to epi $g_{A}$ if and only if $A$ is an interval of even integers. We thus easily get examples of functions which 
are $(\mathbf{Z} \times \mathbf{Z})$-convex as well as examples of functions which are not. The set $C_{I}$ is in general neither an epigraph nor a strict epigraph.

Proposition 4.4. Let $u: E \rightarrow[-\infty,+\infty]$ be a convex function on a vector space $E$. Let $P$ be a subset of $E$. Then the restrictions $\left.\lfloor u\rfloor\right|_{P}$ and $\left.\lceil u\rceil\right|_{P}$ are $(P \times \mathbf{Z})$ convex. In particular $\left.\lceil\operatorname{cvx} g\rceil\right|_{P}$ and $\left.[\widetilde{\widetilde{g}}\rceil\right|_{P}$ are $(P \times \mathbf{Z})$-convex for any function $g: P \rightarrow[-\infty,+\infty] \mathbf{z}$.

Proof. Writing $f=\left.\lfloor u\rfloor\right|_{P}$ and $g=\left.\lceil u\rceil\right|_{P}$ we have (cf. (1.1))

$$
u-1<f \leqslant u \text { and } u \leqslant g<u+1 \text { in } P \text {, }
$$

which implies that $\operatorname{epi}_{\mathrm{s}}(u-1) \cap(P \times \mathbf{Z})=$ epi $f$ and epi $u \cap(P \times \mathbf{Z})=$ epi $g$. Hence the functions $f$ and $g$ are $(P \times \mathbf{Z})$-convex.

Theorem 4.5. Let $E$ be a vector space and $P$ one of its subsets. For any $(P \times \mathbf{Z})$ convex function $f: P \rightarrow \mathbf{Z}$ we have $\operatorname{cvx} f \leqslant\lceil\operatorname{cvx} f\rceil \leqslant f \leqslant \operatorname{cvx} f+1$ in $P$.

Proof. The inequality cvx $f \leqslant f$ holds for any function. Hence cvx $f \leqslant\lceil\operatorname{cvx} f\rceil \leqslant$ $\lceil f\rceil=f$.

For the last inequality we argue as follows. Let $C=\operatorname{cvx}(\operatorname{epi} f)$ and $v=\operatorname{cvx} f$. Then $C \cap(P \times \mathbf{Z})=$ epi $f$ and epi $_{\mathrm{s}} v \subset C \subset$ epi $v$. If $v(p)<q$, then $(p, q) \in C$, which implies that $(p, q) \in$ epi $f$, i.e., $f(p) \leqslant q$. Take now $q=\lceil v(p)+\varepsilon\rceil$, where $\varepsilon>0$. Then $v(p)<q$, so that $f(p) \leqslant\lceil v(p)+\varepsilon\rceil<v(p)+1+\varepsilon$. Letting $\varepsilon$ tend to zero we see that $f(p) \leqslant v(p)+1=(\operatorname{cvx} f)(p)+1$. This completes the proof of the theorem.

We define

$$
P^{j}=\{p \in P ; f(p)=\lceil(\operatorname{cvx} f)(p)\rceil+j\}, \quad j=0,1 .
$$

In view of the last theorem we have $P=P^{0} \cup P^{1}$. We also define

$$
A^{j}=\{p \in P ; f(p)=(\operatorname{cvx} f)(p)+j\}, \quad j=0,1 .
$$

Corollary 4.6. With $f$ as in the theorem, $P$ can be divided into three disjoint sets: $P^{0} \backslash A^{0}, A^{0}$, and $A^{1}=P^{1}$. The first set is precisely the set of points $p$ such that $(\operatorname{cvx} f)(p)$ is not an integer.

Proof. It is clear that the three sets $P^{0} \backslash A^{0}, A^{0}$ and $P_{1}$ are pairwise disjoint. It is also easy to see that $p \in A^{0} \cup A^{1}$ if and only if $(\operatorname{cvx} f)(p)$ is an integer. It follows that $A^{j} \subset P^{j}$. Finally, we shall prove that $P^{1} \subset A^{1}$. If $p \in P^{1}$, then $\lceil(\operatorname{cvx} f)(p)\rceil$ is equal to $f(p)-1$. But we always have $(\operatorname{cvx} f)(p) \geqslant f(p)-1$, so that $(\operatorname{cvx} f)(p)=\lceil(\operatorname{cvx} f)(p)\rceil$ and $p$ belongs to $A^{1}$.

Let us say that a function $u: \mathbf{R}^{n} \rightarrow[-\infty,+\infty]$ is of fast growth if for any constant $c$ the set $\left\{x \in \mathbf{R}^{n} ; u(x) \leqslant c\|x\|_{2}\right\}$ is bounded. The same terminology applies to a function defined in a subset $P$ of $\mathbf{R}^{n}$; we understand that it takes 
the value $+\infty$ outside $P$. In particular, if $f$ is equal to plus infinity outside a bounded set, it is of fast growth.

Theorem 4.7. Let $P$ be a discrete subset of $\mathbf{R}^{n}$ and let $f: P \rightarrow[-\infty,+\infty]_{\mathbf{Z}}$ be a function of fast growth. Then $f$ is $(P \times \mathbf{Z})$-convex if and only if $f=\lceil\operatorname{cvx} f\rceil$, in other words the set $P^{1}$ is empty, and we have

$$
(\operatorname{cvx} f)(p) \leqslant f(p)<(\operatorname{cvx} f)(p)+1, \quad p \in P .
$$

It is equivalent to say that there exists a convex function $u$ such that $f=\lceil u\rceil$.

Proof. We already know from Proposition 4.4 that the condition is sufficient.

To prove necessity, assume that $f$ is $(P \times \mathbf{Z})$-convex. Then $C=\operatorname{cvx}($ epi $f)$ is a convex subset of $\mathbf{R}^{n} \times \mathbf{R}$ such that $C \cap(P \times \mathbf{Z})=$ epi $f$. However, we now know that $C$ is closed, so that actually $C=\operatorname{epicvx} f$. We also know from the previous theorem that $\operatorname{cvx} f \leqslant f \leqslant \operatorname{cvx} f+1$ in $P$. A point $(p, f(p)-1)$ does not belong to epi $f$ and hence not to $C$. Since $C$ is closed and its boundary is defined by $\operatorname{cvx} f$, we must have $f(p)-1<(\operatorname{cvx} f)(p)$, which was to be proved.

We can now take a look again at Examples 4.2 and 4.3 .

Example 4.8. In Example 4.2 we find that

$$
\left(\operatorname{cvx} f_{0}\right)(x)=\widetilde{\widetilde{f}}_{0}(x)=\left(\operatorname{cvx} f_{1}\right)(x)=\widetilde{\widetilde{f}}_{1}(x)=\alpha x, \quad x \in \mathbf{R} .
$$

Thus $\operatorname{cvx} f_{0} \leqslant f_{0}<\operatorname{cvx} f_{0}+1$, but $\left(\operatorname{cvx} f_{0}\right)(0)=0 \neq 1=f_{1}(0)$, so that $f_{1}(0)=\left(\operatorname{cvx} f_{1}\right)(0)+1$. This shows that some condition is necessary in the theorem.

Example 4.9. In Example 4.3 we also have that $\widetilde{\widetilde{g}}_{A}=\operatorname{cvx} g_{A}$. We find that $\left(\operatorname{cvx} g_{A}\right)(x)=\frac{1}{2} x$ for all $x \in \mathbf{R}$ when $A$ is nonempty. Therefore $\left\lceil\operatorname{cvx} g_{A}\right\rceil \neq g_{A}$ if $A$ is nonempty but not equal to all of $2 \mathbf{Z}$. In fact, we then have $\left\lceil\left(\operatorname{cvx} g_{A}\right)(p)\right\rceil=$ $\frac{1}{2} p<g_{A}(p)=\frac{1}{2} p+1$ when $p \in 2 \mathbf{Z} \backslash A$. Still $g_{A}$ is convex if $A$ is an interval of $2 \mathbf{Z}$. When $A$ is empty we have $\left(\operatorname{cvx} g_{\varnothing}\right)(x)=\frac{1}{2} x+\frac{1}{2}$.

Given $u: \mathbf{R}^{n} \rightarrow[-\infty,+\infty]$ we define $u_{r}(x)=u(x)$ if $\|x\|_{2} \leqslant r$ and $u_{r}(x)=$ $+\infty$ otherwise. We also define $u_{[r]}(x)=\max \left(u(x),\|x\|_{2}^{2}-r\right)$. Then $u_{r}$ and $u_{[r]}$ are of fast growth, and we note that $u$ is convex if and only if all the $u_{r}$ are convex, or, equivalently, all the $u_{[r]}$ are convex. The functions $u_{r}$ and $u_{[r]}$ decrease to $u$ as $r$ tends to plus infinity. The same applies to functions $f: P \rightarrow[-\infty,+\infty]$ or $f: P \rightarrow[-\infty,+\infty]_{\mathbf{z}}$.

Corollary 4.10. Let $P$ be a discrete subset of $\mathbf{R}^{n}$ and let a function $f: P \rightarrow$ $[-\infty,+\infty]_{\mathbf{z}}$ be given. Then $f$ is $\left(\mathbf{Z}^{n} \times \mathbf{Z}\right)$-convex if and only if $f_{r}=\left\lceil\operatorname{cvx} f_{r}\right\rceil$ for all $r \in] 0,+\infty\left[\right.$, equivalently if and only if $f_{[r]}=\left\lceil\operatorname{cvx} f_{[r]}\right]$ for all $r \in \mathbf{N}$, where $f_{r}$ and $f_{[r]}$ are defined as before the statement of the corollary.

Proposition 4.11. Let $E$ be a vector space and $P$ any of its subsets. If $f_{j}$, $j \in J$, are $(P \times \mathbf{Z})$-convex functions, then $\sup f_{j}$ is $(P \times \mathbf{Z})$-convex. If the index 
set $J$ is ordered and filtering to the right, and if $\left(f_{j}\right)_{j \in J}$ is a decreasing family of $(P \times \mathbf{Z})$-convex functions, then its infimum inf $f_{j}$ is $(P \times \mathbf{Z})$-convex as well.

Proof. We note that epi $\left(\sup _{j} f_{j}\right)=\bigcap_{j}$ epi $f_{j}$. The latter set is $(P \times \mathbf{Z})$-convex according to Proposition 3.7. Hence $\sup _{j} f_{j}$ is $(P \times \mathbf{Z})$-convex.

For the second part we note that $\operatorname{epi}_{\mathrm{s}}\left(\inf _{j} f_{j}\right)=\bigcup_{j} \operatorname{epi}_{\mathrm{s}} f_{j}$. Now epi $u$ and epi $u$ are convex at the same time, so it follows from Proposition 3.7 that the latter set is $(P \times \mathbf{Z})$-convex. Hence $\operatorname{epi}_{\mathbf{s}}\left(\inf _{j} f_{j}\right)$ is $(P \times \mathbf{Z})$-convex.

\section{Functions Which Are Both Convex and Concave}

A function $u$ such that $-u$ is convex is called concave. A real-valued function on $\mathbf{R}^{n}$ which is both convex and concave is necessarily affine, i.e., of the form $u(x)=\alpha \cdot x+\beta$ for some $\alpha \in \mathbf{R}^{n}$ and $\beta \in \mathbf{R}$. In this section we shall investigate such functions in the discrete case.

Proposition 5.1. Let $P$ be a nonempty subset of a vector space $E$ and $f: P \rightarrow \mathbf{R}$ a real-valued function. Given a linear form $\alpha \in E^{\star}$ and a real number $\beta$ we let $h_{\alpha, \beta}$ be the smallest constant $h \in[0,+\infty]$ such that

$$
0 \leqslant \alpha(p)+\beta \leqslant f(p) \leqslant \alpha(p)+\beta+h, \quad p \in P .
$$

We let $h_{\alpha}=\inf _{\beta \in \mathbf{R}} h_{\alpha, \beta}$ be the smallest constant $h$ such that (5.1) holds for some $\beta \in \mathbf{R}$. Then $h_{\alpha}=\widetilde{f}(\alpha)+\widetilde{g}(-\alpha)$, where for ease in notation we have written $g$ for $-f$. Moreover, $h_{\alpha}=h_{\alpha, \beta}$ for a unique $\beta$, viz. $\beta=-\widetilde{f}(\alpha)$.

Proof. The inequality $\alpha(p)+\beta \leqslant f(p)$ for all $p \in P$ is equivalent to $\widetilde{f}(\alpha) \leqslant-\beta$, and the inequality $f(p)=-g(p) \leqslant \alpha(p)+\beta+h$ for all $p \in P$ is equivalent to $\widetilde{g}(-\alpha) \leqslant \beta+h$. Therefore (5.1) implies that $\widetilde{f}(\alpha)+\widetilde{g}(-\alpha) \leqslant-\beta+(\beta+h)=h$.

Conversely, if $h$ is a real number and $\widetilde{f}(\alpha)+\widetilde{g}(-\alpha) \leqslant h$, then $\widetilde{f}(\alpha)$ is a real number: $\tilde{f}(\alpha)=-\infty$ would imply that $f$ is identically equal to $+\infty$, which is excluded by hypothesis, and $\widetilde{g}(-\alpha)=-\infty$ would imply that $f$ is identically $-\infty$, which is also excluded by hypothesis; finally, the inequality excludes that $\tilde{f}(\alpha)$ is equal to $+\infty$. Therefore $\beta=-\widetilde{f}(\alpha)$ (obviously the best choice of $\beta$ ) yields $\widetilde{f}(\alpha) \leqslant-\beta$ and $\widetilde{g}(-\alpha) \leqslant \beta+h$, which, as already noted, is equivalent to (5.1). The infimum of all real $h$ satisfying (5.1) is equal to the infimum of all real $h$ satisfying $\widetilde{f}(\alpha)+\widetilde{g}(-\alpha) \leqslant h$, which completes the proof.

Proposition 5.2. Let $E$ be a vector space and $P$ a subset such that $\operatorname{cvx} P=E$. Let a real-valued function $f: P \rightarrow \mathbf{R}$ be given, and let $h_{*}=\inf _{\alpha \in E^{\star}} h_{\alpha}$ be the smallest constant such that (5.1) holds for some $\alpha \in E^{\star}$ and some $\beta \in \mathbf{R}$. Assume that $h_{*}$ is finite. Then $-\operatorname{cvx} f-\operatorname{cvx}(-f)$ is constant and equal to $h_{*}$. 
Proof. Let $h$ be a number such that $\alpha+\beta \leqslant f \leqslant \alpha+\beta+h$ in $P$ for some $\alpha \in E^{\star}$ and some $\beta \in \mathbf{R}$. Then

$$
\alpha+\beta \leqslant u \leqslant f \leqslant-v \leqslant \alpha+\beta+h \text { in } P,
$$

where $u=\operatorname{cvx} f$ and $v=\operatorname{cvx}(-f)$. Adding $v$ to all members we obtain

$$
\alpha+\beta+v \leqslant u+v \leqslant f+v \leqslant 0 \leqslant \alpha+\beta+h+v \text { in } P .
$$

We see that $u+v$ is a convex function which is nonpositive in all of $P$, thus also in cvx $P$, which by hypothesis is equal to $E$. But such a function must be constant; let us define $\omega=-(u+v) \geqslant 0$. By the same argument, $v+\alpha$ is a constant $\gamma$. We now have $\gamma+\beta \leqslant-\omega \leqslant 0 \leqslant \gamma+\beta+h$, which shows that $h \geqslant \omega$, and, by taking the infimum over all such $h$, that $h_{*} \geqslant \omega$.

Conversely, we note that $-\omega \leqslant f+\gamma-\alpha \leqslant 0$, thus $\alpha-\gamma-\omega \leqslant f \leqslant \alpha-\gamma$, which shows that $\omega \geqslant h_{\alpha} \geqslant h_{*}$. We conclude that $\omega=h_{*}$.

Theorem 5.3. Let $E$ be a vector space and $P$ a subset of $E$ such that $\operatorname{cvx} P=E$. If both functions $f: P \rightarrow \mathbf{Z}$ and $-f$ are $(P \times \mathbf{Z})$-convex, then $f$ deviates at most by $\frac{1}{2}$ from an affine function: there exist a linear form $\alpha \in E^{\star}$ and constants $\beta, \omega \in \mathbf{R}$ such that

$$
0 \leqslant f(p)-\alpha(p)-\beta \leqslant \omega \leqslant 1, \quad p \in P .
$$

The best constant $\omega$ is equal to the constant $-\operatorname{cvx} f-\operatorname{cvx}(-f)$. Also $(\operatorname{cvx} f)(x)=$ $\alpha(x)+\beta$ and $\operatorname{cvx}(-f)(x)=-\alpha(x)-\beta-\omega$ if $\omega$ is chosen as the smallest possible constant.

Proof. We know from Theorem 4.5 that the two convex functions $u=\operatorname{cvx} f$ and $v=\operatorname{cvx}(-f)$ satisfy

$$
u \leqslant f \leqslant u+1 \text { and } v \leqslant-f \leqslant v+1 \text { in } P .
$$

The functions $u$ and $v$ are real-valued convex functions and possess affine minorants. This implies that $f$ satisfies (5.1) with some finite $h$. From Proposition 5.2 and (5.2) we know that $u+v$ is a constant $-\omega$ and that $h_{*}=\omega$ is the best constant in (5.1) when we are allowed to vary both $\alpha$ and $\beta$.

It remains to be seen that $\omega \leqslant 1$. The first inequality in (5.4) can be rewritten in the notation of the previous proof as

$$
-\omega-\gamma \leqslant f-\alpha \leqslant-\omega-\gamma+1
$$

which shows that $h=1$ is an admissible choice; thus the infimum $h_{*}$ of all such $h$ cannot exceed 1 .

The last statement follows from the inequality

$$
\alpha(p)+\beta \leqslant u \leqslant-v \leqslant \alpha(p)+\beta+\omega, \quad p \in P,
$$

where we now know that $u+v=-\omega$, so that 


$$
\alpha(p)+\beta+v \leqslant-\omega \leqslant 0 \leqslant \alpha(p)+\beta+\omega+v,
$$

which forces $\alpha(p)+\beta+\omega+v$ to be equal to 0 . The proof is complete.

We rewrite the theorem in the most common situation:

Corollary 5.4. If both $f: \mathbf{Z}^{n} \rightarrow \mathbf{Z}$ and $-f$ are $\left(\mathbf{Z}^{n} \times \mathbf{Z}\right)$-convex, then there exist $\alpha \in \mathbf{R}^{n}$ and $\beta \in \mathbf{R}$ such that

$$
0 \leqslant f(p)-\alpha \cdot p-\beta \leqslant \omega, \quad p \in \mathbf{Z}^{n},
$$

where $\omega$ is the constant $-\operatorname{cvx} f-\operatorname{cvx}(-f) \leqslant 1$.

Is it possible to take one of the inequalities in (5.5) strict, like in (4.1)? We shall see that this is not always so.

Example 5.5. In Example 4.2 we see that $\alpha p \leqslant f_{0}(p)<\alpha p+1$, whereas $\alpha p<$ $f_{1}(p) \leqslant \alpha p+1$. In each case we have one strict inequality. Both inequalities are optimal $(\omega=1)$.

Example 5.6. In Example 4.3 we see that

$$
\frac{1}{2} p \leqslant g_{A}(p) \leqslant \frac{1}{2} p+1, \quad p \in \mathbf{Z} .
$$

If $A$ is empty this can be improved to $\frac{1}{2} p+\frac{1}{2} \leqslant g_{\varnothing}(p) \leqslant \frac{1}{2} p+1$. If $A$ is equal to all of $2 \mathbf{Z}$, then we have $\frac{1}{2} p \leqslant g_{2 \mathbf{Z}}(p) \leqslant \frac{1}{2} p+\frac{1}{2}$. Thus in these two cases the graph of $g_{A}$ is contained in a strip of height $\omega=\frac{1}{2}$. In all other cases we see that none of the inequalities in (5.6) can be replaced by a strict inequality. We already remarked above that $g_{A}$ is $(\mathbf{Z} \times \mathbf{Z})$-convex if and only if $A$ is an interval of even numbers. We note that both $g_{A}$ and $-g_{A}$ are $(\mathbf{Z} \times \mathbf{Z})$-convex if and only if $A=\varnothing$ or $A=2 \mathbf{Z}$ or $A$ is a semi-infinite interval.

The example shows that there is a choice between the intervals $[0, \omega[$ and ] $0, \omega]$ in the inequality (5.3) for different values of $p$. This choice is made precise in the following result.

Theorem 5.7. Let $f: \mathbf{Z}^{n} \rightarrow \mathbf{Z}$ and $-f$ be $\left(\mathbf{Z}^{n} \times \mathbf{Z}\right)$-convex and let $\alpha \in \mathbf{R}^{n}$ and $\beta \in \mathbf{R}$ be such that (5.3) holds with $\omega=h_{*}$, i.e., with the smallest $h$ possible. Define

$$
D^{j}=\left\{(p, f(p)) \in \mathbf{Z}^{n} \times \mathbf{Z} ; f(p)=\alpha \cdot p+\beta+j \omega\right\}, \quad j=0,1,
$$

and

$$
A^{j}=\pi_{n+1}\left(D^{j}\right)=\left\{p \in \mathbf{Z}^{n} ; f(p)=\alpha \cdot p+\beta+j \omega\right\}, \quad j=0,1,
$$

where $\pi_{n+1}: \mathbf{Z}^{n} \times \mathbf{Z} \rightarrow \mathbf{Z}^{n}$ denotes the projection which forgets the last coordinate. Assume that $\omega>0$. Then $A^{0}$ and $A^{1}$ are disjoint, and $D^{0}$ and $D^{1}$ are $\left(\mathbf{Z}^{n} \times \mathbf{Z}\right)$ convex. 
Proof. That $A^{0}$ and $A^{1}$ are disjoint follows from the fact that $f$ takes different values in them: $f(p)=\alpha \cdot p+\beta$ when $p \in A^{0}$ while $f(p)=\alpha \cdot p+\beta+\omega$ if $p \in A^{1}$.

The sets $T^{j}=\left\{(p, q) \in \mathbf{Z}^{n} \times \mathbf{Z} ; q=\alpha \cdot p+\beta+j \omega\right\}$ are $\left(\mathbf{Z}^{n} \times \mathbf{Z}\right)$-convex, as are epi $f$ and hypo $f$. Therefore so are the intersections $D^{0}=T^{0} \cap$ epi $f$ and $D^{1}=T^{1} \cap$ hypo $f$.

\section{Digital Hyperplanes}

The concept of naive discrete line was introduced by Reveillès (1991:48). Such a line is defined to be the set of all integer points $p \in \mathbf{Z}^{2}$ such that $0 \leqslant$ $\alpha_{1} p_{1}+\alpha_{2} p_{2}<\max \left(\left|\alpha_{1}\right|,\left|\alpha_{2}\right|\right)$, where $\alpha_{1}$ and $\alpha_{2}$ are relatively prime integers. Generalizing this slightly, we define a naive digital hyperplane as the set of all points $p \in \mathbf{Z}^{n}$ which satisfy the double inequality

$$
0 \leqslant \alpha \cdot p+\beta<h
$$

for some $\alpha \in \mathbf{R}^{n} \backslash\{0\}$ and some $\beta \in \mathbf{R}$, where $h=\|\alpha\|_{\infty}$. We remark that one can always interchange the strict and the non-strict inequalities: the set just defined can equally well be defined by

$$
0<(-\alpha) \cdot p-\beta-\omega \leqslant h .
$$

The precise size of $h$ is important for the representation of the hyperplane as the graph of a function of $n-1$ variables as shown by the following result.

Theorem 6.1. Define

$$
T=\left\{p \in \mathbf{Z}^{n} ; 0 \leqslant \alpha \cdot p+\beta \leqslant h\right\} \text { and } T_{\mathrm{s}}=\left\{p \in \mathbf{Z}^{n} ; 0<\alpha \cdot p+\beta<h\right\},
$$

where $\alpha \in \mathbf{R}^{n} \backslash\{0\}, \beta \in \mathbf{R}$ and $h>0$, and let

$$
T^{j}=\left\{p \in \mathbf{Z}^{n} ; \alpha \cdot p+\beta=j h\right\}, \quad j=0,1 .
$$

Let $D$ be a subset of $\mathbf{Z}^{n}$ which is contained in $T$ and contains $T_{\mathrm{s}}$ and define $D_{\mathrm{s}}=D \cap T_{\mathrm{s}}$ and $D^{j}=D \cap T^{j}$. Fix an integer $k=1, \ldots, n$ and let $\pi_{k}: \mathbf{Z}^{n} \rightarrow$ $\mathbf{Z}^{n-1}$ be the projection which forgets the $k^{\text {th }}$ coordinate. Then $\left.\pi_{k}\right|_{D}$ is injective if $h<\left|\alpha_{k}\right|$, and $\left.\pi_{k}\right|_{D}$ is surjective if $h>\left|\alpha_{k}\right|$. If $h=\left|\alpha_{k}\right|$, then $\left.\pi_{k}\right|_{D}$ is injective if and only if $\pi_{k}\left(D^{0}\right)$ and $\pi_{k}\left(D^{1}\right)$ are disjoint, and $\left.\pi_{k}\right|_{D}$ is surjective if and only if $\pi_{k}\left(D^{0} \cup D^{1}\right)=\pi_{k}\left(T^{0} \cup T^{1}\right)$.

Proof. For ease in notation we let $k=n$ and write $p^{\prime}=\left(p_{1}, \ldots, p_{n-1}\right)$ and similarly for $\alpha$. Then $p$ belongs to $T$ if and only if

$$
-\alpha^{\prime} \cdot p^{\prime}-\beta \leqslant \alpha_{n} p_{n} \leqslant-\alpha^{\prime} \cdot p^{\prime}-\beta+h,
$$

and $p$ belongs to $T_{\mathrm{s}}$ if and only if

$$
-\alpha^{\prime} \cdot p^{\prime}-\beta<\alpha_{n} p_{n}<-\alpha^{\prime} \cdot p^{\prime}-\beta+h .
$$


Clearly for every $p^{\prime}$ there is at most one $p_{n}$ which satisfies the inequalities if $h<\left|\alpha_{n}\right|$ or if $h=\left|\alpha_{n}\right|$ and $\left(\alpha^{\prime} \cdot p^{\prime}+\beta\right) / h$ is not an integer. Also there is at least one $p_{n}$ if $h>\left|\alpha_{n}\right|$ or if $h=\left|\alpha_{n}\right|$ and $\left(\alpha^{\prime} \cdot p^{\prime}+\beta\right) / h$ is not an integer. Here it does not matter whether we use (6.3) or (6.4), so the conclusion holds also for $D$.

The case when $h=\left|\alpha_{n}\right|$ and $\left(\alpha^{\prime} \cdot p^{\prime}+\beta\right) / h$ is an integer remains to be considered. Then we see that there are two values of $p_{n}$ which satisfy $(6.3)$ and none that satisfies (6.4). Hence there is at most one $p_{n}$ such that $\left(p^{\prime}, p_{n}\right)$ belongs to $D=D^{0} \cup D_{\mathrm{s}} \cup D^{1}$ if and only if $\pi_{k}\left(D^{0}\right)$ and $\pi_{k}\left(D^{1}\right)$ are disjoint. There is at least one $p_{n}$ such that $\left(p^{\prime}, p_{n}\right)$ belongs to $D$ if and only if $\pi_{k}\left(D^{0} \cup D^{1}\right)$ contains every point in the projection of $T^{0} \cup T^{1}$. This completes the proof.

We do not suppose here that $h=\|\alpha\|_{\infty}$. However, this is the most natural case: we then know that $\left.\pi_{k}\right|_{D}$ is a bijection for any $k$ such that $\left|\alpha_{k}\right|=\|\alpha\|_{\infty}$ and the conditions on the $D^{j}$ are satisfied, and that $\left.\pi_{j}\right|_{D}$ is surjective for all $j$ such that $\left|\alpha_{j}\right|<\|\alpha\|_{\infty}$.

In view of Theorems 5.7 and 6.1 it seems reasonable to propose the following definition.

Definition 6.2. A refined digital hyperplane is a $\mathbf{Z}^{n}$-convex subset $D$ of $\mathbf{Z}^{n}$ which is contained in $T$ and contains $T_{\mathrm{s}}$, where $T$ and $T_{\mathrm{S}}$ are the slabs defined by (6.1) for some $\alpha \in \mathbf{R}^{n} \backslash\{0\}, \beta \in \mathbf{R}$, and $h>0$; and in addition is such that, for at least one $k$ such that $\left|\alpha_{k}\right|=h$, the sets $D^{j}=D \cap T^{j}$ have disjoint projections $\pi_{k}\left(D^{j}\right)$, and $\pi_{k}\left(D^{0} \cup D^{1}\right)=\pi_{k}\left(T^{0} \cup T^{1}\right)$.

The naive hyperplanes now appear as a special case, viz. when $D^{0}=T^{0}$, and $D^{1}$ is empty, or conversely, and $\left|\alpha_{k}\right|=\|\alpha\|_{\infty}$.

Example 6.3. Define $D=\left(D^{0} \times\{0\}\right) \cup\left(D^{1} \times\{1\}\right)$, where $D^{j}, j=0,1$, are two subsets of $\mathbf{Z}^{n-1}$ such that $D^{1}=\mathbf{Z}^{n-1} \backslash D^{0}$. Then $D$ is a refined digital hyperplane if and only if both $D^{0}$ and $D^{1}$ are $\mathbf{Z}^{n-1}$-convex.

Example 6.4. Define $D=\left\{\left(p_{1}, p_{1}\right) \in \mathbf{Z}^{2} ; p_{1} \leqslant 0\right\} \cup\left\{\left(p_{1}, p_{1}+1\right) \in \mathbf{Z}^{2} ; p_{1} \geqslant 0\right\}$. This is a refined digital hyperplane with $\left|\alpha_{1}\right|=\left|\alpha_{2}\right|=\|\alpha\|_{\infty}=1$. The projection $\pi_{1}$ satisfies the requirements in the definition, but $\pi_{2}$ does not.

The following result motivates the definition just given and relates it to the digitally convex functions we have introduced.

Theorem 6.5. A subset $D$ of $\mathbf{Z}^{n}$ is a refined digital hyperplane if and only if it is the graph of a function $f: \mathbf{Z}^{n-1} \rightarrow \mathbf{Z}$ such that both $f$ and $-f$ are $\left(\mathbf{Z}^{n-1} \times \mathbf{Z}\right)$ convex.

Proof. Let $f$ be a $\left(\mathbf{Z}^{n-1} \times \mathbf{Z}\right)$-convex function such that also $-f$ is $\left(\mathbf{Z}^{n-1} \times \mathbf{Z}\right)$ convex. Then $D=\operatorname{graph} f$ is a refined digital hyperplane according to Theorem 5.7 .

Conversely, if $D$ is a refined digital hyperplane and $h=\left|\alpha_{n}\right|$, then the projection $\left.\pi_{n}\right|_{D}$ is bijective, and this allows us to define a function $f: \mathbf{Z}^{n-1} \rightarrow \mathbf{Z}$, $f\left(p^{\prime}\right)=-\alpha^{\prime} \cdot p^{\prime}-\beta+j h$ as in the proof of Theorem 6.1 with $j=0$ or 1 being uniquely determined by the requirements on the $D^{j}$. This function as well 
as its negative are $\left(\mathbf{Z}^{n-1} \times \mathbf{Z}\right)$-convex, since both its epigraph and its hypograph are $\mathbf{Z}^{n}$-convex. To wit, assuming $\alpha_{n}$ to be positive, its epigraph is equal to $D+(\{0\} \times \mathbf{N})$, and its hypograph is equal to $D+(\{0\} \times(-\mathbf{N}))$.

\section{Conclusions}

In this paper we have studied a simple definition of convex sets in $\mathbf{Z}^{n}$ and of convex functions defined on $\mathbf{Z}^{n}$ and having integer values. The definitions are actually given not only for functions defined on $\mathbf{Z}^{n}$ but for other subsets of $\mathbf{R}^{n}$ as well.

We have shown that the functions so defined share important properties of convex functions defined on vector spaces, viz. concerning the relation between convex sets and convex functions, and suprema and infima of families of functions. We have also clarified how much a convex digital function can deviate from a convex function of real variables.

From several points of view the definitions seem to be satisfying. They are extremely simple and easy to grasp; nevertheless, there are nontrivial difficulties in checking whether a given function is convex.

A kind of ambiguity in the values of a convex function is shown to be inevitable: in general a convex function is not determined by its restriction to the complement of a point.

Functions that are both convex and concave are of interest as candidates for defining digital hyperplanes; in fact we have shown that they define sets which are precisely the sets satisfying a refined definition of digital hyperplanes.

Acknowledgment. I am grateful to Erik Melin for comments on earlier versions of the manuscript, to Jean-Pierre Reveillès for clarifying the history of the notion of a naive digital line, and to an anonymous referee for help with references.

\section{References}

Eckhardt, Ulrich 2001. Digital lines and digital convexity. Lecture Notes in Computer Science 2243, pp. 209-228.

Hiriart-Urruty, Jean-Baptiste; Lemaréchal, Claude 2001. Fundamentals of Convex Analysis. Springer-Verlag. X +259 pp.

Hörmander, Lars 1994. Notions of Convexity. Boston: Birkhäuser. viii +414 pp.

Melin, Erik 2003. Digital straight lines in the Khalimsky plane. Uppsala University, Department of Mathematics, Report 2003:30. Accepted for publication in Mathematica Scandinavica.

Reveillès, J[ean]-P[ierre] 1991. Géométrie discrète, calcul en nombres entiers et algorithmique. Strasbourg: Université Louis Pasteur. Thèse d'État, 251 pp.

Rosenfeld, Azriel 1974. Digital straight line segments. IEEE Transactions on Computers. c-32, No. 12, 1264-1269.

Rosenfeld, Azriel; Klette, Reinhard 2001. Digital straightness. Electronic Notes in Theoretical Computer Science 46, 32 pp. http://www.elsevier.nl/ locate/entcs/volume 46 .html

Singer, Ivan 1997. Abstract Convex Analysis. John Wiley \& Sons, Inc. xix +491 pp. 